\title{
On Parity in Diatomic Molecules and Application of a Rigorous Algorithm for the Prediction of Nitric Oxide Spectra
}

\author{
James 0 Hornkohl ${ }^{1}$ and Christian G Parigger ${ }^{2 *}$ \\ ${ }^{1}$ Hornkohl Consulting, 344 Turkey Creek Road, Tullahoma, TN, USA \\ ${ }^{2}$ University of Tennessee, University of Tennessee Space Institute, Center for Laser Applications, Tullahoma, TN, USA
}

Received: March 01, 2017; Accepted: March 23, 2017; Published: March 24, 2017

*Corresponding author: Christian Parigger, Associate Professor, University of Tennessee, University of Tennessee Space Institute, Center for Laser Applications, 411 B.H. Goethert Parkway, Tullahom, TN 37388-9700, USA, Tel: (931)393-7338/509; E-mail: cparigge@tennessee.edu

\section{Abstract}

Application of the parity operator to the general diatomic eigenfunction reveals that the parity eigenvalue is a product of two factors, one that depends on the total angular momentum quantum number and a second constant factor which can be interpreted as the intrinsic parity of the molecule. These new results allow one to rigorously design an algorithm for the computation of diatomic spectra by utilizing that allowed transitions have non-vanishing rotational line strength factors.

Keywords: Parity; Molecular spectroscopy; Diatomic molecules;

\section{Introduction}

The diatomic Hamiltonian matrix is historically paritypartitioned, thereby giving parity a more important role in diatomic spectroscopy than in atomic spectroscopy, e.g., see Zare, et al [1]. Typically, each spectral line in a highly resolved diatomic spectrum is labeled with the e/f parity designation of the lower level [2] in addition to the branch designation and the value of the J quantum number for the lower level. Several authors, for instance Hougen [3], Røeggen [4], Judd [5] and Larsson [6], have presented treatments of diatomic parity using the Born-Oppenheimer approximate separation of the diatomic eigenfunction into rotational, vibrational and electronic factors. They also used atomic or molecular orbital models of the electronic eigenfunction to determine its parity. An exact formula relating the parity eigenvalue, $p= \pm 1$, to the total angular momentum quantum number, $\mathrm{J}$, is needed.

In this work, the standard framework of parity and angular momentum methods are applied. The effect of parity on the prediction of heteronuclear diatomic molecular spectra is presented. Subsequently, the algorithm for the computation of molecular spectra is described. As a specific example, results for nitric oxide spectra are compared with experimental data.

\section{Methods}

Operation of the parity operator on the general WignerWitmer [7] diatomic eigenfunction is used to yield the parity eigenvalues that are composed of a constant and an angular momentum dependent part. The computation of diatomic molecular spectra is accomplished without the need of explicitly including parity selection rules. The fundamental Wigner-Witmer diatomic eigenfunction simplifies the determination of rotational line strengths.

\section{Parity operator}

The discrete parity operation can be accomplished with a rotation and a reflection. The parity operator, $\mathrm{P}$ can be written as a product,

$$
\mathrm{P}=\mathrm{C}_{2} \sigma_{v} \text {. }
$$

The determinant of the matrix representations $\sigma_{v}(y, z)$ and $\mathrm{C}_{2}(x)$ in laboratory xyz-coordinates are -1 and +1 , respectively. The $\mathrm{C}_{2}$ operator is a proper rotation that can be expressed as a discrete transformation of Euler angles. The Euler angles are the arguments of the Wigner $D$-function used to formulate rotational symmetry, consequently, the eigenvalues of $\mathrm{C}_{2}$ are controlled by the angular momentum, $J$. The $\sigma_{v}(y, z)$ operation results in a constant factor, and the $\mathrm{C}_{2}$ operation yields the angular momentum dependent part of the parity eigenvalue.

\section{Rotation operator and Wigner D-function}

Molecular eigenfunctions are normally expressed in rotated coordinates. The representations of the eigenfunction in original and rotated coordinate systems are connected with the rotation operator, $\mathrm{R}(\alpha, \beta, \gamma)$ Euler angles $\alpha, \beta$ and $\gamma$ and the Wigner $D$-functions,

$$
\begin{aligned}
& \left\langle\mathbf{r}_{1}, \mathbf{r}_{2}, \ldots, \mathbf{r}_{\mathrm{N}} \mid J M\right\rangle=\sum_{\Omega=-J}^{J}\left\langle\mathbf{r}_{1}, \mathbf{r}_{2}, \ldots, \mathbf{r}_{\mathrm{N}}|\mathrm{R}(\alpha, \beta, \gamma)| J \Omega\right\rangle\left\langle J \Omega\left|\mathrm{R}^{\dagger}(\alpha, \beta, \gamma)\right| J M\right\rangle \\
& =\sum_{\Omega=-J}^{J}\left\langle\mathbf{r}_{1}^{\prime}, \mathbf{r}_{2}^{\prime}, \ldots, \mathbf{r}_{\mathrm{N}}^{\prime} \mid J \Omega\right\rangle D_{M \Omega}^{J^{*}}(\alpha, \beta, \gamma) .
\end{aligned}
$$


Angular momentum is the generator of rotations, therefore, one can expect that application of the discrete operator $\mathrm{C}_{2}$ to the arguments of the $D$ function would yield a relationship between angular momentum and parity. In terms of spatial and angular coordinates of diatomic molecules, Equation (2) can be written as

$$
\begin{aligned}
& \left\langle\rho, \zeta, \chi, \mathbf{r}_{2}, \ldots, \mathbf{r}_{n}, \mathrm{r}, \theta, \varphi \mid n v J M\right\rangle \\
& =\sum_{\Omega=-J}^{J}\left\langle\rho, \zeta, \chi^{\prime}, \mathbf{r}_{2}^{\prime}, \ldots, \mathbf{r}_{N}^{\prime}, \mathrm{r}, \theta^{\prime}, \varphi^{\prime} \mid n v J \Omega\right\rangle D_{M \Omega}^{J^{*}}(\alpha, \beta, \gamma) .
\end{aligned}
$$

Here $\rho$ is the distance of one electron (the electron arbitrarily labeled 1 but it could be any one of the electrons), $\zeta$ is the distance of that electron above or below the plane that passes through the center of mass of the two nuclei (the coordinate origin), and $\chi$ is the angle of rotation of that electron about the internuclear vector $\mathbf{r}(\mathrm{r}, \theta, \varphi)$ The vibrational quantum number $v$, has been extracted from the quantum numbers collection, $n$, which represents all required quantum numbers except $J, M$ $\Omega$ and $v$.

The variables $\rho, \zeta$ and $\mathrm{r}$ are scalars, unaffected by rotations. The physical rotation $\theta$ and the angle of coordinate rotation $\alpha$ are also about the same axis, namely the first intermediate $y$ axis of the full coordinate rotation. The angles $\chi$ and $\gamma$ are both rotations about the $z^{\prime}$ axis. Thus,

$\varphi^{\prime}=\varphi-\alpha, \theta^{\prime}=\theta-\beta, \chi^{\prime}=\chi-\gamma$.

In coordinate rotations, one is at liberty to choose $\alpha, \beta$ and $\gamma$. If one chooses for the angles $\alpha=\varphi, \beta=\theta$ and $\gamma=\chi$, all angular dependence of $\left\langle\rho, \zeta, \chi^{\prime}, \mathbf{r}_{2}^{\prime}, \ldots, \mathbf{r}_{N}^{\prime}, \mathbf{r},{ }^{\prime}, \varphi^{\prime} \mid n v J \Omega\right\rangle$ is removed. This yields the Wigner and Witmer [7] diatomic eigenfunction,

$$
\begin{aligned}
& \left\langle\rho, \zeta, \chi, \mathbf{r}_{2}, \ldots, \mathbf{r}_{n}, \mathrm{r}, \theta, \varphi \mid n J M\right\rangle= \\
& \sum_{\Omega=-J}^{J}\left\langle\rho, \zeta, \mathbf{r}_{2}^{\prime}, \ldots, \mathbf{r}_{N}^{\prime}, \mathrm{r} \mid n v\right\rangle D_{M \Omega}^{J^{*}}(\varphi, \theta, \chi) .
\end{aligned}
$$

The values of the quantum numbers, $J$ and $\Omega$ influence the electronic-vibrational eigenfunction $\left\langle\rho, \zeta, \mathbf{r}_{2}^{\prime}, \ldots, \mathbf{r}_{N}^{\prime}, r \mid n v\right\rangle$, but the electronic-vibrational eigenfunction is not an angular momentum state vector.

\section{Results \\ Parity of diatomic states}

Parity is rotationally invariant. Inversion of the signs of all rotated coordinates inverts the signs of all un-rotated coordinates, and vice versa. Therefore, the parity operator can be represented by $\mathrm{C}_{2}\left(x^{\prime}\right) \sigma_{v}\left(y^{\prime}, z^{\prime}\right)$. The application to the right side of the Wigner-Witmer diatomic eigenfunction (5) yields the parity eigenvalues,

$$
\begin{aligned}
& p=-p_{\Sigma}(-1)^{J} \quad J: \text { half }- \text { integer, } \\
& p=p_{\Sigma}(-1)^{J} \quad J: \text { integer. }
\end{aligned}
$$

The constant part of the parity eigenvalue, $p_{\Sigma}$ is labeled in accord with standard spectroscopic notation. The imaginary values of $(-1)^{\mathrm{J}}$ occurring when $J$ is half-integer can be avoided if one adopts the convention [2] to always subtract $1 / 2$ from $J$ when $J$ is half-integer. With this convention, Eq. (6) is replaced by

$$
p=-p_{\Sigma}(-1)^{J-1 / 2} \quad J: \text { half }- \text { integer. }
$$

The value of $p_{\Sigma}$ does not depend upon quantum numbers. It is a global value applying to all states of a given molecule. If the diatomic molecule can be said to have an intrinsic parity, then it is clearly $p_{\Sigma}$. One would expect the product of the intrinsic parities of the fundamental particles composing the molecule to equal $p_{\Sigma}$.

\section{Parity in an algorithm for computing diatomic spectra}

The following describes an algorithm in which Eqs. (7) and (8) become practical equations for computing diatomic parity.

Consider the algorithm for computation of the wavelengths and intensities in the spectrum of a molecule from the first principles of quantum mechanics. The upper $H^{\prime}$ and lower $H$ Hamiltonian matrices are computed and numerically diagonalized by unitary matrices, $U^{\prime}$ and $U$. The upper $F_{n^{\prime} v^{\prime} J^{\prime}}^{\prime}$ and lower $F_{n v s}$ terms are the eigenvalues of the Hamiltonians,

$$
\begin{aligned}
& F_{n^{\prime} v^{\prime} J^{\prime}}^{\prime}=U^{\prime \dagger} H^{\prime} U^{\prime} \\
& F_{n v J}=U^{\dagger} H U,
\end{aligned}
$$

and the vacuum wavenumbers, $\widetilde{v}$, of the predicted spectral lines,

$$
\tilde{v}=F_{n^{\prime} v^{\prime} J^{\prime}}^{\prime}-F_{n v J}
$$

are term differences. Of the very large number of computed term differences only those for which the Condon and Shortley [8] line strength does not vanish are spectral lines. The line strength, $S\left(n v J, n^{\prime} v^{\prime} J^{\prime}\right)$, is the sum over all $M$ and $M^{\prime}$ of the irreducible tensor $T_{k}^{(q)}$ expectation values, $\left\langle n v J M\left|T_{k}^{(q)}\right| n^{\prime} v^{\prime} J^{\prime} M^{\prime}\right\rangle$. The exact separation of the total angular momentum in the WignerWitmer diatomic eigenfunction results in a diatomic line strength composed of two parts,

$$
S\left(n v J, n^{\prime} v^{\prime} J^{\prime}\right)=S\left(n v, n^{\prime} v^{\prime}\right) S\left(J, J^{\prime}\right),
$$

the electronic-vibrational strength, $S\left(n v, n^{\prime} v^{\prime}\right)$ and the unitless rotational line strength or Hönl-London factor, $S\left(J, J^{\prime}\right)$. The Born-Oppenheimer approximation separates the electronicvibrational strength into electronic and vibrational parts. In the Hund's case (a) basis built from the Wigner-Witmer eigenfunction, the third Euler angle, $\chi=\gamma$,appears in the Wigner $D$-function,

$$
|a\rangle=|n v J M \Omega S \Sigma\rangle=\sqrt{\frac{2 J+1}{8 \pi^{2}}}\left\langle\rho, \zeta, \mathbf{r}_{2}^{\prime}, \ldots, \mathbf{r}_{N}^{\prime}, \mathbf{r} \mid n v\right\rangle|S \Sigma\rangle D_{M \Omega}^{J^{*}}(\varphi, \theta, \chi)
$$


The algorithm for computation of the vacuum wavenumbers, $\widetilde{v}$ and line strengths, $S\left(n v J, n^{\prime} v^{\prime} J^{\prime}\right)$, of diatomic spectral lines is a straightforward application of quantum mechanics, but except for the very simplest molecules is also very far removed from the realm of the possible. However, with two very stringent caveats, the algorithm can be implemented for the diatomic molecule. The first caveat is that the vacuum wavenumbers, $\widetilde{v}$, for many spectral lines in many bands of a band system must have been experimentally measured with high accuracy such as that provided by Fourier transform spectroscopy. Secondly, using semi-empirical molecular constants one must be able to build upper and lower Hamiltonian matrices whose eigenvalue differences accurately predict the measured vacuum wavenumbers. A fitting process is required [1]. One assumes trial values for the molecular constants, computes the spectral lines positions, $\widetilde{v}$, and from the differences between $\tilde{v}-\tilde{v}_{\text {exp }}$ finds the corrections to the molecular parameters. The difference between computed and measured line positions will typically equal the measurement error margins.

The Hund's case (a) basis is mathematically complete. A sum of basis functions, $|a\rangle$, can be quantitatively very accurate. The parity operator, $\mathrm{P}$, commutes with the Hamiltonian. Thus, the orthogonal matrix that diagonalizes the case (a) representation of the Hamiltonian will also diagonalize the case (a) representation of $\mathrm{P}$.

\section{Application to the prediction of nitric oxide spectra}

As a specific example, the line position data of Faris and Cosby [10] are used for the NO beta $(3,0)$ band for then purpose of creating a complete line list for the band with line strengths. Figure 1 illustrates a spectrum generated from the NO line list.

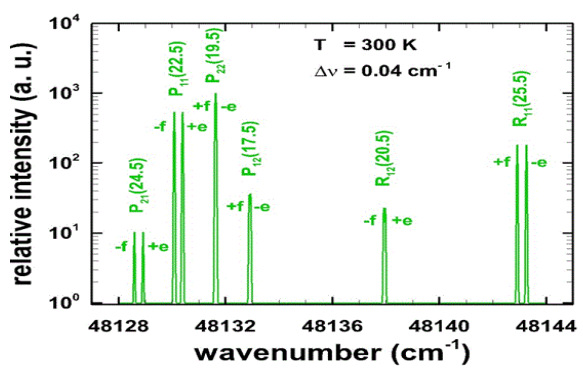

Figure 1: Section of computed spectrum of the NO $B^{2} \Pi-X^{2} \Pi(3,0)$ band. Also see Table 1.

A multiphoton $1+1$ excitation was used to observe 10 of the 12 possible branches [10], with particular attention to the parity designations of the numerous $\Lambda$ doublets. These data are particularly suited for testing applications of the algorithm for the calculation of diatomic spectra. A total of 428 lines were fitted with a standard deviation of $0.030 \mathrm{~cm}^{-1} \mathrm{~cm}$, and a line list having no missing lines for the range of upper and lower $J$ values was computed. Table 1 provides details of the lines displayed in Figure 1. The computed parity eigenvalues agree with those assigned by
Faris and Cosby [10].

Table 1: Lines of the NO $B^{2} \Pi-X^{2} \Pi(3,0)$ band, see Fig. 1. The Hönl-London factors, $S\left(J, J^{\prime}\right)$, and parity eigenvalues, $p$, are derived from numerical diagonalization of Hamiltonians in Hund's case (a) basis. The $P_{22}(19.5) \Lambda$ doublet is not resolved in the experiments [10].

\begin{tabular}{|c|c|c|c|c|c|c|c|}
\hline$J$ & & $p$ & $F_{J^{\prime}}$ & $F_{J}$ & $\widetilde{v}$ & $S\left(J, J^{\prime}\right)$ & $\widetilde{v}-\widetilde{v}_{\text {e } p}$ \\
\hline & & & $\left(\mathrm{cm}^{-1}\right)$ & $\left(\mathrm{cm}^{-1}\right)$ & $\left(\mathrm{cm}^{-1}\right)$ & & $\left(\mathrm{cm}^{-1}\right)$ \\
\hline 24.5 & $P_{21}$ & $-f$ & 49111.456 & 982.866 & 48128.590 & 0.813 & 0.021 \\
\hline 24.5 & $P_{21}$ & $+e$ & 49111.501 & 982.588 & 48128.914 & 0.812 & 0.078 \\
\hline 24.5 & $P_{11}$ & $-f$ & 48952.618 & 822.538 & 48130.080 & 21.749 & 0.014 \\
\hline 24.5 & $P_{11}$ & $+e$ & 48952.673 & 822.282 & 48130.390 & 21.805 & -0.020 \\
\hline 24.5 & $P_{22}$ & $+f$ & 48876.118 & 744.504 & 48131.615 & 18.832 & -0.007 \\
\hline 24.5 & $P_{22}$ & $-e$ & 48876.146 & 744.505 & 48131.641 & 18.786 & \\
\hline 24.5 & $P_{12}$ & $+f$ & 48747.194 & 614.306 & 48132.888 & 0.515 & -0.013 \\
\hline 24.5 & $P_{12}$ & $-e$ & 48747.244 & 614.307 & 48132.936 & 0.527 & -0.012 \\
\hline 24.5 & $R_{12}$ & $-f$ & 48952.618 & 814.699 & 48137.919 & 0.881 & 0.034 \\
\hline 24.5 & $R_{12}$ & $+e$ & 48952.673 & 814.701 & 48137.971 & 0.880 & -0.077 \\
\hline 24.5 & $R_{11}$ & $+f$ & 49210.930 & 1068.021 & 48142.908 & 25.424 & 0.034 \\
\hline 24.5 & $R_{11}$ & $-e$ & 49210.985 & 1067.731 & 48143.254 & 25.317 & -0.018 \\
\hline
\end{tabular}

\section{Discussion}

The exact separation of the coordinates of the total angular momentum in the Wigner $D$-function greatly simplifies implementation of the algorithm which uses non-vanishing line strengths to determine if a computed term difference represents an allowed spectral line. The Hönl-London factors are computed from the Hund's case (a) transition moment and the matrices, $U$ and $U^{\prime}$, which diagonalize the upper and lower Hamiltonians.

A single selection rule handles all types of diatomic spectra. If the Hönl-London factor, $S\left(J, J^{\prime}\right)$ is non-vanishing, then the transition is allowed. Parity plays no part in the fitting process which determines the molecular parameters, but the parity eigenvalues are computed from the finalized values of the molecular parameters. The presented algorithm can be used to predict molecular spectra for the purpose of fitting measured data [9].

\section{Conclusions}

The essence of this work clearly is the theoretical simplification for the prediction of diatomic molecular spectra. Extensive parity considerations that are presented in classic texts on molecular spectroscopy appear to not be necessary, rather the straightforward application of the Wigner-Witmer function allows one to conclude that a transition is allowed if the rotational line strength is non-vanishing. Parity is not utilized in the determination of the molecular parameters, consequently, the computation and analysis of diatomic molecular spectra is significantly alleviated. 


\section{Acknowledgments}

One of us (CGP) acknowledges support in part by the Accomplished Center of Excellence, Center for Laser Application at The University of Tennessee, University of Tennessee Space Institute. In addition, the outstanding dedication of late James o. Hornkohl towards diatomic molecular spectroscopy is greatly appreciated during the last 30 years.

\section{References}

1. Zare RN, Schmeltekopf AL, Harrop WJ, Albritton DL. A direct approach for the reduction of diatomic spectra to molecular constants for the construction of RKR potentials. J Mol Spectrosc. 1973;46(1):37-66.

2. Brown JM, Hougen JT, Huber KP, Johns JWC, Kopp I, Lefebvre-Brion H, et al. The labeling of parity doublet levels in linear molecules. J Mol Spectrosc. 1975;55(1-3):500-503.

3. Hougen JT. The Calculation of Rotational Energy Levels and Rotational Line Intensities in Diatomic Molecules. National Institute of Standards and Technology: Boulder, CO. 2001.

4. Røeggen I. The inversion eigenvalues of non- $\sigma$ states of diatomic molecules, expressed in terms of quantum numbers. Theor Chim Acta. 1971;21(4):398-409. doi:10.1007/BF00528562.

5. Judd B. Angular Momentum Theory for Diatomic Molecules. Academic Press: New York, NY. 1975.

6. Larsson M. Phase Conventions for Rotating Diatomic Molecules. Phys Scr. 1981;23(5A):835-836.

7. Wigner E, Witmer EE. On the structure of the diatomic molecular spectra according to quantum mechanics. Z Phys. 1928;51:859-886. Hettema $\mathrm{H}$, editor. On the structure of the spectra of two-atomic molecules according to quantum mechanics. Quantum Chemistry: Classic Scientific Papers. World Scientific: Singapore. 2000;287-311. doi: 10.1142/9789812795762_0018.

8. Condon EU, Shortley GH. The Theory of Atomic Spectra. Cambridge Univ. Press, Cambridge, UK. 1953.

9. Parigger CG, Woods AC, Surmick DM, Gautam G, Witte MJ, Hornkohl JO. Computation of diatomic molecular spectra for selected transitions of aluminum monoxide, cyanide, diatomic carbon, and titanium monoxide. Spectrochim Acta Part B At Spectrosc. 2015;107:132-138.

10.Faris GW, Cosby PC. Observation of $N O \quad B^{2} \Pi(v=3) \leftarrow X^{2} \Pi(v=0)$ absorptions with 1+1 multiphoton ionization: Precision line position measurements and parity assignment of the $B^{2} \Pi$ state. J Chem Phys. 1992;97(10):7073-7086. 